Médiation du discours expert : approches linguistiques

\title{
Michel Van der Yeught, Manuel d'anglais de la bourse et de la finance
}

Paris : Éditions Ophrys, collection Langues de spécialité, 2013

\section{Marc Eline}

\section{(2) OpenEdition \\ Journals}

\section{Édition électronique}

URL : http://journals.openedition.org/asp/4173

DOI : 10.4000/asp.4173

ISSN : 2108-6354

\section{Éditeur}

Groupe d'étude et de recherche en anglais de spécialité

\section{Édition imprimée}

Date de publication : 1 mars 2014

Pagination : 134-137

ISSN : 1246-8185

Référence électronique

Marc Eline, « Michel Van der Yeught, Manuel d'anglais de la bourse et de la finance », ASp [En ligne], 65 | 2014, mis en ligne le 01 mars 2014, consulté le 02 novembre 2020. URL : http:// journals.openedition.org/asp/4173; DOI : https://doi.org/10.4000/asp.4173

Ce document a été généré automatiquement le 2 novembre 2020.

Tous droits réservés 


\section{Michel Van der Yeught, Manuel d'anglais de la bourse et de la finance}

Paris : Éditions Ophrys, collection Langues de spécialité, 2013

Marc Eline

\section{RÉFÉRENCE}

Van der Yeught, Michel. 2013. Manuel d'anglais de la bourse et de la finance. Paris : Éditions Ophrys, collection Langues de spécialité. 283 pages. ISBN 978-2-7080-1366-7. 
1 Ce manuel complète, par un apport pédagogique, l'ouvrage récent de recherche consacré à l'anglais de la bourse et de la finance, publié dans la collection Langues de spécialité dirigée par Michel Van der Yeught aux éditions Ophrys en partenariat avec le GERAS et le LERMA (EA 853) d'Aix-Marseille Université.

2 L'objectif de M. Van der Yeught, spécialiste d'anglais financier et auteur d' Une histoire de Wall Street (2009), est de proposer aux étudiants en économie, gestion et banque-finance inscrits en cursus de licence ou de master une véritable plateforme de formation - voire d'autoformation - à la langue de spécialité

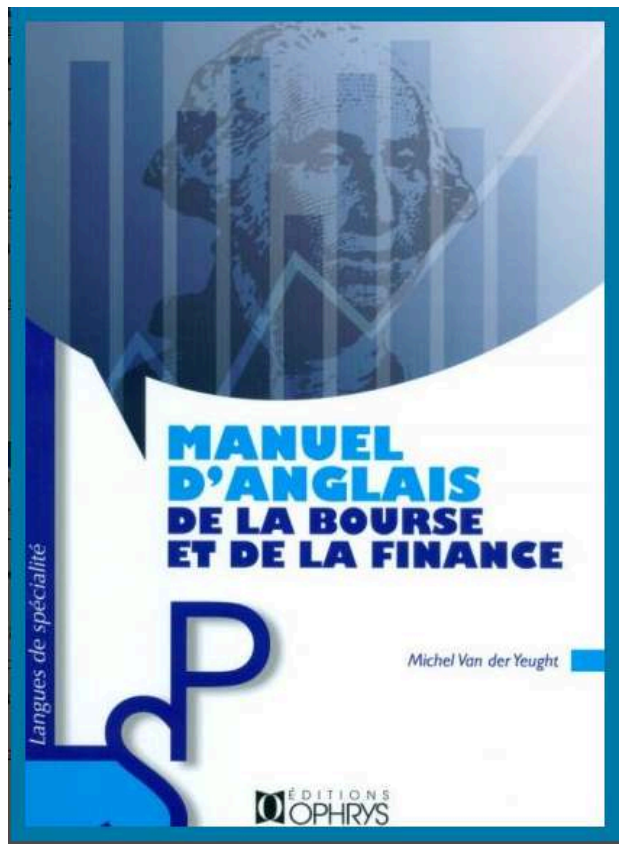
qu'ils doivent maîtriser tant dans leurs études que dans l'environnement professionnel dans lequel ils souhaitent travailler.

3 La première partie, riche en contenu linguistique et culturel, est une introduction aux quatre domaines retenus : " the financial environment ", " the stock market ", " major stock exchanges in Britain and America » et enfin « accounting ». L'ensemble est présenté sous la forme de vingt-six dossiers thématiques qui englobent des notions et concepts essentiels à la compréhension du monde de la finance et de la bourse tels que l'environnement réglementaire, la banque commerciale et la banque d'affaires, les marchés d'action et les marchés obligataires, les acquisitions boursières et l'organisation des principales bourses du Royaume-Uni et des États-Unis. L'auteur propose une définition des nombreux concepts abordés, rappelle l'origine historique d'institutions ou d'appellations que chacun connaît, comme Wall Street, la compagnie pétrolière Esso ou l'audit. L'accent fort mis sur le contenu technique et sur la terminologie spécialisée n'exclut ni la richesse linguistique, avec des formules d'argumentation ou des formes grammaticales utiles reprises en gras, ni une mise en perspective qui souligne les évolutions et tendances observées sur les marchés. Le sujet des bourses et des marchés boursiers britanniques et américains, qui occupe la moitié des dossiers, se voit accorder une place de choix. L'étudiant en banque-finance y trouvera matière à comprendre leur fonction dans le financement de l'économie et à s'interroger sur les apports et sur les limites de l'innovation financière qui a donné naissance aux produits dérivés, que Warren Buffett, le "sage d'Omaha ", qualifiait d'armes de destruction massive (2002 : 15). Un rappel historique sur les origines et les avatars de la City, du London Stock Exchange (LSE) et du New York Stock Exchange (NYSE) éclaire le lecteur sur le rôle crucial joué par la bourse dans le développement de l'industrie aux $19^{\mathrm{e}}$ et $20^{\mathrm{e}}$ siècles, ainsi que sur les parties prenantes, des entreprises en quête de financements non bancaires, aux organismes de supervision et de régulation, en passant par les fonds d'investissement et les agences de notation telles que Moody's et Standard \& Poor's. Celles-ci font l'objet d'un dossier spécifique qui reflète leur importance pour les investisseurs et émetteurs, entreprises et États y compris. M. Van 
der Yeught souligne leur rôle controversé dans le développement des crises financières récentes, qu'il s'agisse des subprimes ou de la crise grecque, qu'elles sont soupçonnées d'avoir précipitées par des révisions aussi tardives que brutales de leurs avis. Comme le rappelle Howard Davies (2010), ancien directeur de la London School of Economics and Political Science, elles opèrent en premier lieu en dépit d'un conflit d'intérêts patent dans la mesure où elles sont rémunérées par les émetteurs dont elles évaluent la qualité de la « signature ». Pire encore, dans le cas des titrisations qui regroupent des volumes importants de dettes à mettre sur le marché, elles conseillent contre due rémunération les émetteurs sur la meilleure façon de structurer ces opérations connues pour leur opacité, qu'elles vont ensuite "noter", alors même qu'elles maîtrisent très mal le risque associé à ces émissions d'un type nouveau. Le dernier volet de cette première partie du manuel est consacré à la comptabilité anglo-saxonne, domaine technique souvent négligé dans les manuels d'anglais de l'économie ou de la finance; on sait pourtant qu'une connaissance même rudimentaire des états financiers de type anglais ou américains et des référentiels (GAAP, IFRS) s'impose dès lors qu'on veut analyser la situation financière d'une entreprise, comprendre l'articulation entre le bilan, les résultats et le cours boursier d'un groupe ou encore mesurer avec pertinence la solidité d'une institution financière, dont certaines, jugées «too big to fail », n'ont pourtant pas survécu à la crise. L'auteur de ce manuel l'a bien compris, qui a su dépasser le simple cadre lexical pour s'intéresser aux principes qui sous-tendent les choix d'enregistrement des transactions ou d'interprétation des opérations. Chaque dossier est ainsi assorti de documents issus de la presse, de sites professionnels ou de l'administration, illustrant un aspect essentiel du domaine, comme la valorisation au prix de marché («mark-to-market») connue pour ses effets dans la crise des subprimes, ou encore les "dark pools", terme souvent directement emprunté à l'anglais et qui désigne des plateformes boursières privées dont l'activité, relativement nouvelle, reste opaque pour l'investisseur lambda, voire pour les autorités de tutelle. Dans un souci pédagogique, un tableau lexical bilingue, un jeu de questions et une brève netographie permettent d'éclairer la compréhension de chaque dossier et d'engager des recherches et une réflexion préalables à un débat ou à des exposés. Par ailleurs, tous les dossiers comportent plusieurs paragraphes d'entraînement au thème, axés sur le lexique propre au dossier et complétés par un corrigé partiel fort utile. Quelques redites, probablement intentionnelles car de nature transversale, facilitent le cas échéant une lecture indépendante ou non séquentielle des dossiers. Les connaissances mises à la disposition du lecteur, la richesse qu'apporte la langue de spécialité dans l'approche, que celle-ci soit terminologique, technique, culturelle ou historique, confèrent à cet ouvrage une portée qui dépasse le public étudiant initialement mentionné pour s'étendre à l'enseignant de langue de spécialité (ou encore de finance responsable d'un cours en anglais), au chercheur intéressé par le monde de la finance anglo-saxonne, voire au praticien opérant sur le marché domestique et désireux de perfectionner son anglais en vue de se reconvertir sur les marchés internationaux ou de s'affranchir d'un certain «plafond de verre ». Mis en appétit par le dossier présentant les activités des professionnels de la bourse, on pourra éventuellement regretter pour les étudiants en cycle de master qu'il ne comprenne pas un volet décrivant les métiers, les responsabilités par poste et les défis spécifiques au recrutement et aux carrières de la banque d'affaires et de marché. On notera cependant que l'auteur attire l'attention du lecteur sur les questions sociétales récurrentes et pertinentes de la rémunération des opérateurs de marché ou des banquiers que soulève l'attribution des bonus ou des stock 
options et dont la presse se fait régulièrement l'écho en critiquant leur excès au regard de la valeur ajoutée créée ou tout simplement des revenus du reste de la population.

La deuxième partie comprend un ensemble de ressources pédagogiques. En premier lieu, deux études de cas en anglais financier sont proposées: la première, la plus simple, concerne la situation de la société Hewlett-Packard, qui porte le nom de ses deux fondateurs issus de l'université de Stanford. Préalablement à l'exposé des attentes exigibles (description de la société, analyse simple de la situation financière et de ses causes, diagnostic général et recommandations), l'auteur dispense de nombreux conseils à visée pédagogique permettant de rendre l'exercice intéressant et abordable, ainsi que de le dupliquer à d'autres entreprises, sous réserve de faire un choix judicieux de sociétés. Les suggestions destinées à l'enseignant portent aussi bien sur les secteurs ou le type de structure, que sur le choix du corpus à étudier et sur les supports pertinents, lesquels incluent par exemple aux côtés de la presse ou des états financiers les rapports d'analystes ou les graphiques boursiers. La phase de préparation et les modalités pratiques de cet exercice d'étude de cas qui peut paraître technique et même déroutant aux yeux d'un professeur de langue sont décrites en détail, dans un souci louable de facilitation et d'opérationnalité de la démarche pour l'enseignant et les étudiants. Une seconde étude de cas, plus complexe, porte sur la société Apple à la fin de l'exercice 2012 et sera exploitée dans les mêmes conditions, mutatis mutandis. Dans les deux cas, un corpus diversifié et des pistes d'analyse sont fournis de sorte que l'étude puisse être exploitée en l'état.

5 Le reste de l'ouvrage, soit la fin de la deuxième partie et le troisième et dernier chapitre, fournit au lecteur une "trousse à outils» lexicale et grammaticale, dans laquelle on retrouve les verbes irréguliers usuels, mais aussi 200 expressions anglaises figurant dans les dossiers, expliquées et assorties d'exemples repris en contexte bancaire ou financier. L'intérêt opérationnel de cette liste est d'avoir regroupé les expressions, non par ordre alphabétique, mais par type de fonction discursive dans un environnement professionnel peuplé de managers et autres "decision makers»; ainsi pourra-t-on repérer aisément des verbes ou qualificatifs utiles pour structurer une analyse, décrire une situation problématique, exprimer une opinion ou des sentiments, formuler une proposition et des décisions, et tirer une conclusion résumant l'issue d'un problème. Enfin, des rappels sur l'expression des nombres et sommes d'argent, une liste de sigles et d'abréviations courantes en banque et en finance, un lexique anglaisfrançais, un index et une bibliographie-filmographie indicative viennent clore les nombreuses ressources pédagogiques de l'ouvrage.

6 En conclusion, il s'agit là d'un manuel qui présente l'intérêt d'un contenu riche sur le plan technique, culturel et historique, souvent complexe, mais rendu abordable grâce à des explications et des illustrations très claires. Il apporte la preuve, s'il en était encore besoin, que la langue de spécialité doit être abordée en tenant compte du milieu dont elle est le reflet, des enjeux et normes qui contraignent ce milieu, de son histoire et de sa culture. Les sujets couverts et la méthode pédagogique, tantôt explicative ou descriptive, tantôt socratique, invitant au questionnement, assurent, à notre sens, un solide socle de connaissances en anglais financier. Certes, la mathématisation croissante des concepts, applications et produits financiers pourrait justifier d'étendre à l'avenir cet ouvrage à la langue de spécialité des experts de la finance de marché, qui manient les "grecques ", outils de mesure du risque et de la volatilité et le langage des probabilités et du calcul stochastique, mais on risquerait de se situer aux confins de 
l'anglais de la finance et de celui des mathématiques et probabilités. Il reste que cet ouvrage remplit très efficacement, à notre sens, sa fonction première, qui est de permettre au lecteur de s'approprier progressivement l'anglais de la finance et de la bourse et de s'assurer, ce faisant, la crédibilité et la légitimité requises au regard des attentes et exigences élevées du monde professionnel de la banque et de la finance.

\section{BIBLIOGRAPHIE}

Buffett, Warren. 2003. Letter to the shareholders of Berkshire Hathaway Inc. Berkshire Hathaway.com. <http://www.berkshirehathaway.com/letters/2002pdf.pdf>. Consulté le 16 janvier 2014.

Davies, Howard. 2010. The Financial Crisis: Who is to Blame? Cambridge, UK : Polity Press.

Van der Yeught, Michel. 2009. Une histoire de Wall Street. Paris : Éditions ESKA.

\section{AUTEURS}

\section{MARC ELINE}

Université Panthéon-Assas Paris 2. marc.eline@u-paris2.fr 